\title{
Pedicle Corridors and Vessel Options for Free Flap Reconstruction following Endoscopic Endonasal Skull Base Surgery: A Systematic Review
}

\author{
Rajan P. Dang ${ }^{1}$ Lauren T. Roland ${ }^{2,3}$ Jeffrey D. Sharon ${ }^{3}$ Michelle Doering ${ }^{4}$ Michael R. Chicoine ${ }^{5}$ \\ Patrik Pipkorn ${ }^{1}$
}

${ }^{1}$ Department of Otolaryngology-Head and Neck Surgery, Washington

University in St Louis, Saint Louis, Missouri, United States

${ }^{2}$ Department of Otolaryngology-Head and Neck Surgery, Emory

University, Atlanta, Georgia, United States

${ }^{3}$ Department of Otolaryngology-Head and Neck Surgery, University

Address for correspondence Patrik Pipkorn, MD, Department of Otolaryngology-Head and Neck Surgery, Washington University School of Medicine, 660 South Euclid Avenue, Campus Box 8057, St. Louis, MO 63110, United States (e-mail: pipkornp@ent.wust.edu).

of California, San Francisco, California, United States

${ }^{4}$ Department of Otolaryngology-Head and Neck Surgery,

Washington University School of Medicine, Saint Louis,

Missouri, United States

${ }^{5}$ Department of Neurosurgery, Washington University in St Louis,

Saint Louis, Missouri, United States

J Neurol Surg B 2021;82:196-201.

\begin{abstract}
Introduction Microvascular free flaps offer an alternative to local and regional flaps for coverage of complex or large skull base defects. Routes and approaches to these reconstructive options are complicated and require an understanding of complex head and neck anatomy.

Methods A systematic review of the literature was performed using a set of search terms with the help of a qualified librarian. Articles were reviewed and selected for inclusion based on relevance. We were interested in reporting possible routes for free flap accessibility to the skull base as well as microvascular vessel options, as this choice may affect the geometry and accessibility to the defect.

Results A total of 1,917 articles were obtained from a comprehensive search and 11 articles were ultimately found to be relevant to this review. Published options for vessel

\section{Keywords}

- endoscopic

- skull base

- free flap

- reconstruction anastomosis and corridors to the skull base following endoscopic endonasal surgery are reviewed, including Caldwell-Luc/transbuccal space, prevertebral space, transpterygoid/parapharyngeal, and transmaxillary approaches.

Conclusion The field of endoscopic surgery has continued to advance and provide options for tumors of the skull base. This has led to a need for creative routes to the skull base for free flap reconstruction.
\end{abstract}

received

March 19, 2019

accepted after revision

July 3, 2019

published online

September 12, 2019 (c) 2019. Thieme. All rights reserved.

Georg Thieme Verlag KG,

Rüdigerstraße 14

70469 Stuttgart, Germany
DOI https://doi.org/

10.1055/s-0039-1695001. ISSN 2193-6331. 


\section{Introduction}

As endoscopic endonasal skull base surgery has gained momentum, the need for vascularized options for reconstruction of the skull base has become apparent. While the workhouse of reconstruction options for the skull base, the nasoseptal flap (NSF), is a popular and straightforward option, ${ }^{1}$ multiple other pedicled and vascularized options may be more appropriate in certain situations, such as when an NSF has failed, in the setting of septal trauma, or when a larger reconstruction is needed. These options include the pedicled buccal flap, pericranial flap, and temporoparietal fascia flap. ${ }^{1}$

However, there are rare situations in which vascularized tissue larger than those from pedicled options is needed, such as in cases of recurrent cerebrospinal fluid (CSF) leaks, large defects following cancer resections, or compromised bone from skull base osteomyelitis or osteoradionecrosis. In addition, in settings where local options are exhausted or have been exposed to radiation, nonradiated tissue may need to be recruited to achieve a safe skull base reconstruction. ${ }^{1}$ Microvascular free flaps may offer appropriate coverage in these complex situations. Minimally invasive approaches without external scarring are preferred; however, a safe and feasible route for the free tissue transfer pedicle is required. In this review, we discuss published options for corridors for free flap transposition as well as vessel choices for microvascular anastomosis.

\section{Methods}

\section{Search Strategy}

The published literature was searched using strategies created by a medical librarian (M.D.) for surgical flaps for reconstruction of skull base following endoscopic endonasal surgery. The search strategies were established using a combination of standardized terms and keywords and were implemented in Ovid Medline 1946, Embase 1947, Scopus 1960, Cochrane Central Register of Controlled Trials, Cochrane Database of Systematic Reviews, and Clinicaltrials. gov. All searches were completed in January 2019. Duplicates were identified and excluded. Each unique citation was then screened by abstract and title. The full text of each remaining article was reviewed, and additional relevant articles were identified through references.

\section{Quality and Risk of Bias}

The Methodological Index for Non-Randomized Studies (MINORS) criteria was used to assess study quality and risk of bias. ${ }^{2}$ The eight essential components of the MINORS criteria were applied to each study: (1) clearly stated aim, (2) inclusion of consecutive patients, (3) prospective data collection, (4) appropriate endpoints, (5) unbiased assessment of endpoint, (6) appropriate follow-up length, (7) loss to follow-up less than $5 \%$, and (8) prospective calculation of study size. Study quality and risk of bias were reviewed independently by two authors (R.P.D. and P.P.).

\section{Results}

\section{Study Selection}

Results were exported to Endnote for a total of 1,917 results. A total of 968 duplicates were assumed to be accurately identified and removed for a total of 949 unique citations. A total of 932 articles were excluded after screening by abstract and title, with 15 articles remaining: 7 of these articles were excluded after review of the full text, with 8 articles meeting criteria following systematic review. Three additional relevant articles were identified through references. Consequently, a total of 11

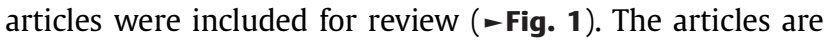
summarized in - Table 1.

\section{Quality and Heterogeneity}

Study quality as assessed by the MINORS criteria is shown in -Table 2. The median score was 4 out of a possible 16 points. The minimum score was 4 and the maximum score was 9 . The studies consisted entirely of cadaver dissections, case reports, and case series; no study included prospectively gathered data or prospectively calculated sample size.

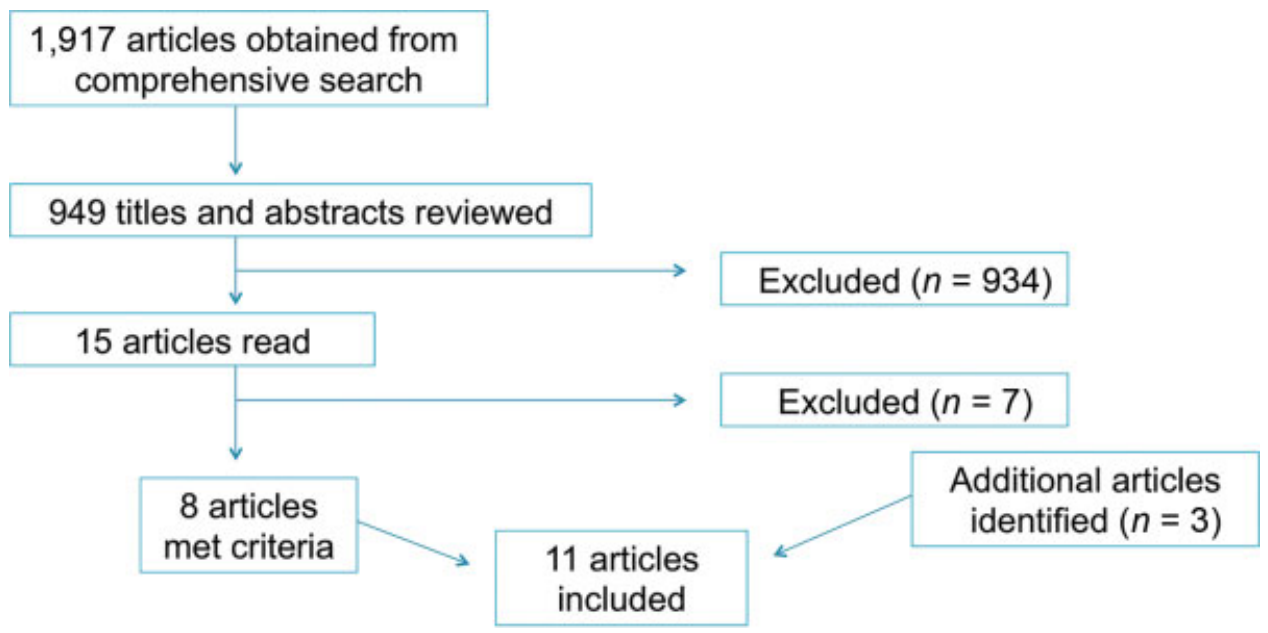

Fig. 1 Flow diagram for selection of studies. 
Table 1 Summary of included articles

\begin{tabular}{|c|c|c|c|c|c|}
\hline Study & $\begin{array}{l}\text { Study } \\
\text { design }\end{array}$ & $\begin{array}{l}\text { No. of patients } \\
\text { (or specimens if } \\
\text { cadaveric study) }\end{array}$ & Technique described & $\begin{array}{l}\text { Described } \\
\text { recipient sites }\end{array}$ & $\begin{array}{l}\text { Potential } \\
\text { recipient sites }\end{array}$ \\
\hline Kamal et al ${ }^{3}$ & Cadaver & 2 & Caldwell-Luc/Buccal space corridor & $\mathrm{C} / \mathrm{F}, \mathrm{PS}$ & \multirow{4}{*}{$\begin{array}{l}\text { C/F, CL, MCF, } \\
\text { O, PS, PT, S }\end{array}$} \\
\hline Hackman $^{4}$ & Case report & 1 & Caldwell-Luc/Buccal space corridor & $\mathrm{CL}$ & \\
\hline Sinha et al ${ }^{5}$ & Case series & 5 & Caldwell-Luc/Buccal space corridor & C/F, PS, S & \\
\hline Kang et $\mathrm{al}^{6}$ & Case series & 4 & Caldwell-Luc/Buccal space corridor & $\mathrm{C} / \mathrm{F}, \mathrm{PS}$ & \\
\hline Durmaz et $\mathrm{al}^{7}$ & Cadaver & 10 & Prevertebral corridor & $\mathrm{CL}, \mathrm{CS}$ & \multirow[t]{2}{*}{$\mathrm{CL}, \mathrm{CS}$} \\
\hline London et $\mathrm{al}^{8}$ & Case report & 1 & Prevertebral corridor & $\mathrm{CL}, \mathrm{CS}$ & \\
\hline $\begin{array}{l}\text { Rivera-Serrano } \\
\text { et } \mathrm{al}^{9}\end{array}$ & Cadaver & 6 & $\begin{array}{l}\text { Transpterygoid/ } \\
\text { Parapharyngeal corridor }\end{array}$ & $\begin{array}{l}\text { "Entire ventral } \\
\text { skull base" }\end{array}$ & \multirow[t]{2}{*}{$\begin{array}{l}\text { C/F, CL, CS, } \\
\text { MCF, O, S }\end{array}$} \\
\hline Krane et al ${ }^{10}$ & Case report & 1 & $\begin{array}{l}\text { Transpterygoid/ } \\
\text { Parapharyngeal corridor }\end{array}$ & $\mathrm{CL}, \mathrm{CS}$ & \\
\hline Kato et al ${ }^{11}$ & Case report & 1 & $\begin{array}{l}\text { Transmaxillary approach with } \\
\text { contralateral neck vessels }\end{array}$ & $\begin{array}{l}\text { "Anterior } \\
\text { skull base" }\end{array}$ & $\mathrm{C} / \mathrm{F}$ \\
\hline Assam et $\mathrm{al}^{12}$ & Cadaver & 17 & $\begin{array}{l}\text { Characterization of maxillary } \\
\text { artery third segment }\end{array}$ & $\mathrm{N} / \mathrm{A}$ & $\mathrm{C} / \mathrm{F}$ \\
\hline Reyes et al ${ }^{13}$ & Cadaver & 1 & $\begin{array}{l}\text { Use of superior trochlear system } \\
\text { as recipient vessels }\end{array}$ & $C / F, P S, S$ & $C / F, P S, S$ \\
\hline
\end{tabular}

Abbreviations: C/F, cribriform/fovea; CL, clivus; CS, upper C-spine; MCF, middle cranial fossa; O, orbit; PS, planum sphenoidale; PT, posterior table frontal sinus; $S$, sella.

Published options for vessel anastomosis and corridors to the skull base are described later.

\section{Caldwell-Luc/Buccal Space Approach for Free Tissue Transfer}

Kamal et al first described a Caldwell-Luc approach for endoscopic reconstruction of the skull base using a radial forearm free flap in cadavers in 2012. ${ }^{3}$ Hackman later described the use of the Caldwell-Luc combined with a transbuccal corridor to the facial vessels to allow access for appropriate free flap placement to cover the clivus following sequestrectomy from osteoradionecrosis. ${ }^{4}$ In this case report, a forearm free tissue transfer was achieved with placement of the pedicle tunneled through a Caldwell-Luc opening into the maxillary sinus. The anterior face of the maxillary wall was removed to allow easy passage for the pedicle. A subcutaneous tunnel through the buccal space was then created between the Caldwell-Luc corridor and the neck. This approach and corridor has also been published in a series of patients with recurrent CSF leaks from our own institution. ${ }^{5}$ Furthermore, Kang et al published a case series in 2018 in which four patients were reconstructed using a similar approach with a vastus lateralis free flap following failure of prior local reconstruction following neoplastic resection (-Fig. 2). ${ }^{6}$

\section{Prevertebral Approach for Free Tissue Transfer}

In a cadaver study, prevertebral approach for tissue transfer was performed 20 times. ${ }^{7}$ In this article, the procedure is performed by mobilizing the carotid sheath anteromedially and creating a plane between the prevertebral fascia and the constrictor musculature until access to the clivus is achieved. London et al described using a prevertebral approach in a case report in which a patient with a significant CSF leak and clival defect due to osteoradionecrosis was reconstructed with a radial forearm free flap. ${ }^{8}$ A Penrose drain was passed through the prevertebral space from the neck to the defect over the clivus to facilitate tunneling of the free flap, which was then placed directly over the clival defect with the pedicle in the neck (-Figs. 3-4).

\section{Transpterygoid/Parapharyngeal Approach for Free Tissue Transfer}

The transparapharyngeal/transpterygoid pathway for skull base reconstruction was first described in a cadaver study for use with pedicled flaps. ${ }^{9}$ In this cadaver study, the tunnel was initially created from the neck by dissection into the parapharyngeal space. The medial pterygoid muscle was identified at the pterygomasseteric sling at the angle of the mandible. By careful dissection along the medial aspect of the medial pterygoid muscle, the lower aspect of the pterygoid plates could be reached. The second part was done endoscopically through a transpterygoid approach. The posterior maxillary wall was removed and the internal maxillary artery was ligated. The pterygoid plates were drilled away as to allow appropriate space for the flap and pedicle transposition. A recent case report describes a similar approach in a patient with granulomatosis with polyangiitis who developed nasopharyngeal destruction and resultant clival and cervical spine osteomyelitis. An anterior serratus free flap was delivered into the nasopharynx transorally, and the lateral nasopharyngeal wall was opened under transnasal endoscopic visualization by creating a tunnel transcervically through the parapharyngeal space. The vascular pedicle of the flap was then delivered into the neck (- Fig. 5). ${ }^{10}$ 

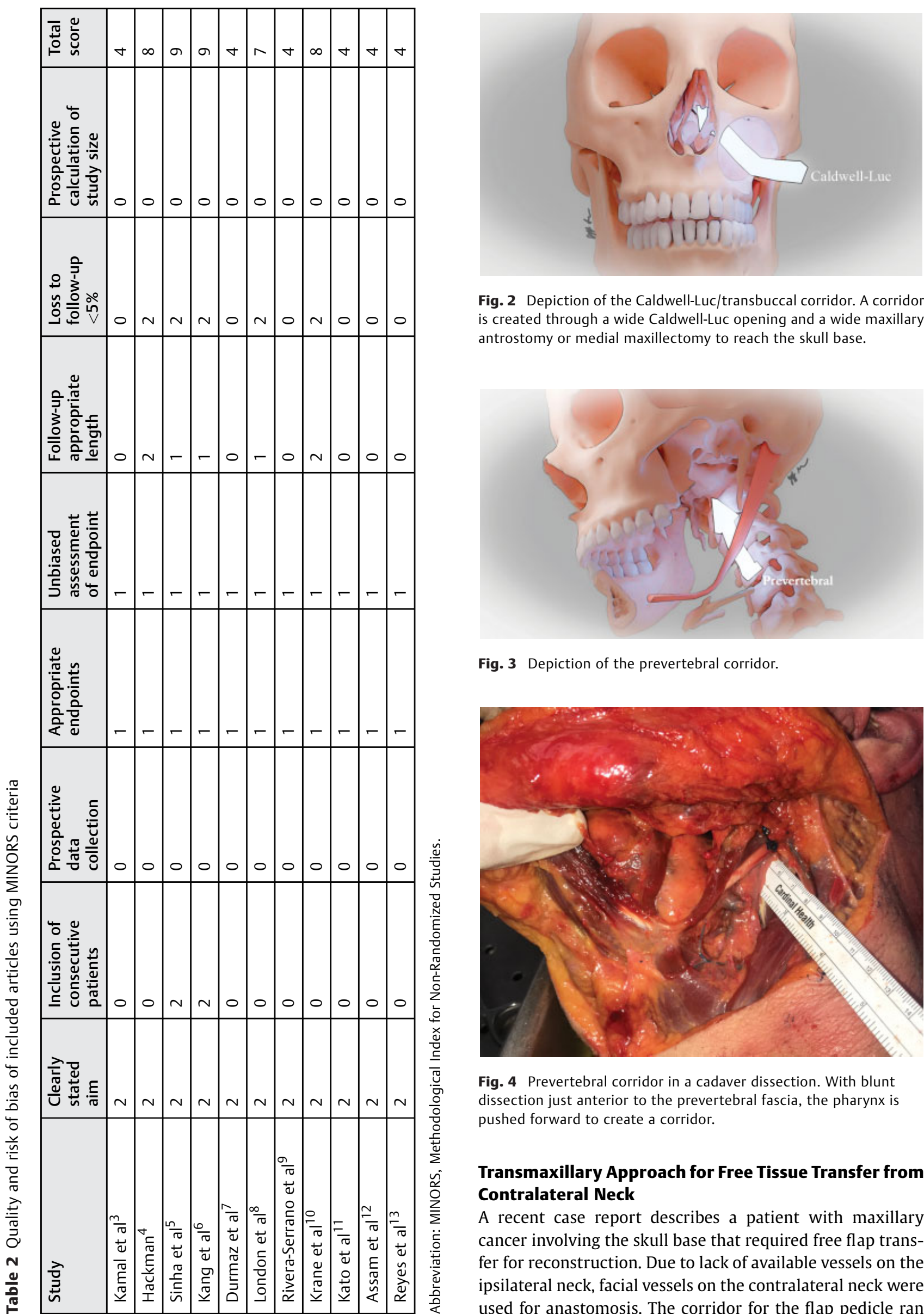

Fig. 2 Depiction of the Caldwell-Luc/transbuccal corridor. A corridor is created through a wide Caldwell-Luc opening and a wide maxillary antrostomy or medial maxillectomy to reach the skull base.

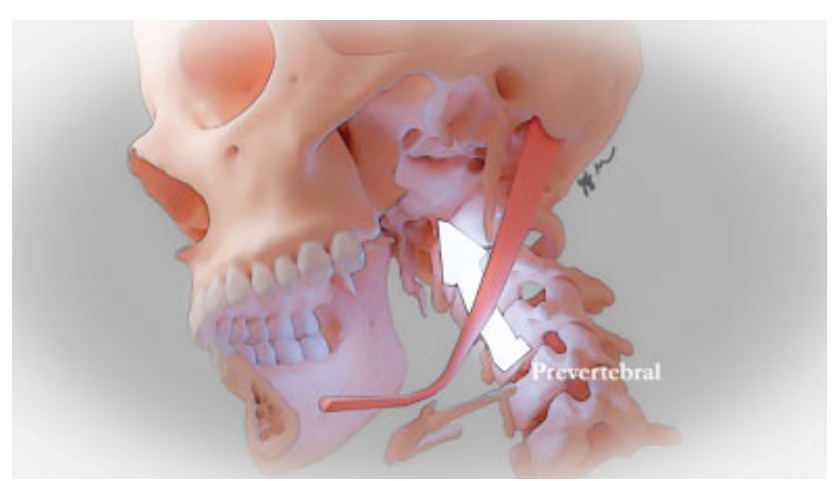

Fig. 3 Depiction of the prevertebral corridor.

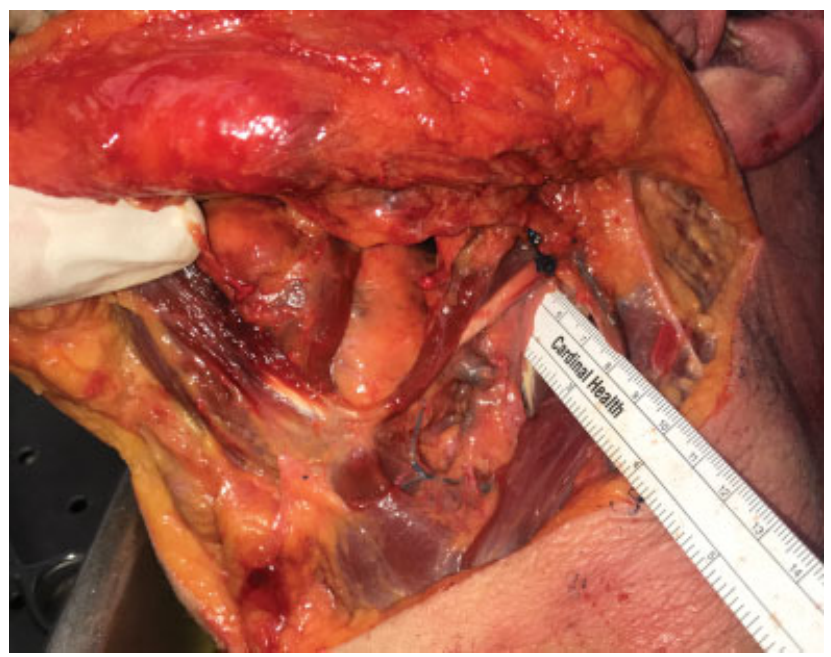

Fig. 4 Prevertebral corridor in a cadaver dissection. With blunt dissection just anterior to the prevertebral fascia, the pharynx is pushed forward to create a corridor.

\section{Transmaxillary Approach for Free Tissue Transfer from Contralateral Neck}

A recent case report describes a patient with maxillary cancer involving the skull base that required free flap transfer for reconstruction. Due to lack of available vessels on the ipsilateral neck, facial vessels on the contralateral neck were used for anastomosis. The corridor for the flap pedicle ran 


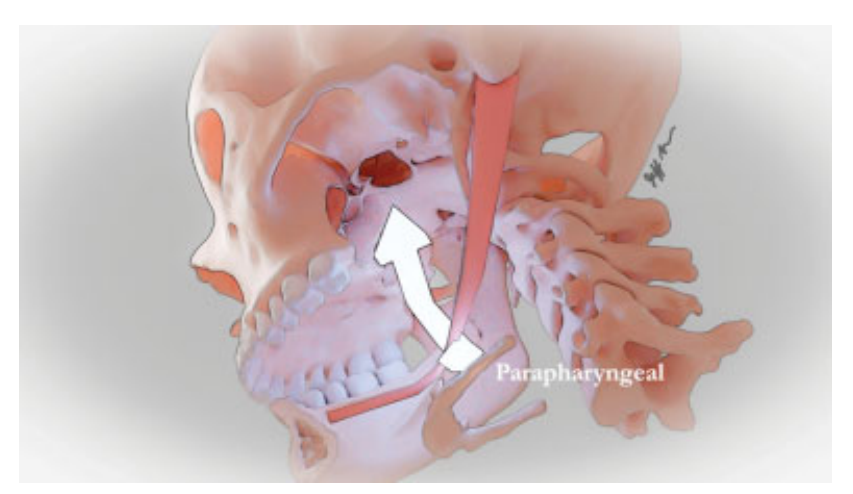

Fig. 5 Depiction of the transpterygoid/parapharyngeal corridor. This corridor is created after the pterygoid plate has been removed. With blunt dissection in the parapharyngeal space, a route for a vascular pedicle is created.

across the subcutaneous area of the maxillary gingiva to reach the inferior border of the contralateral mandible and required $10 \mathrm{~cm}$ of length. ${ }^{11}$ Additional proposed pathways by the authors included a Caldwell-Luc approach $(7 \mathrm{~cm})$ and a corridor involving a medial maxillary wall fenestration with a pathway to the contralateral auricle for superficial temporal artery anastomosis $(10 \mathrm{~cm}){ }^{11}$

\section{Vessel Options for Microvascular Anastomosis}

Vessel options for microvascular anastomosis include the branches of the external carotid in the neck, with the ipsilateral facial vessels being those most commonly used; other options include contralateral facial vessels, the superficial temporal artery, or the superior thyroid artery. ${ }^{8,10}$ In a cadaver study with 17 specimens, Assam et al described the use of the third segment of the maxillary artery as a potential recipient vessel for free flap reconstruction of the midface or skull base. ${ }^{12}$ In another cadaver study, Reyes et al described the use of a fascia lata free flap anastomosed to the superior trochlear system for reconstruction of the skull base. ${ }^{13}$ The recipient vessels were identified via a Lynch incision, and the fascia lata free flap was introduced through the nose. An osteotomy was made at the radix to anastomose the flap pedicle to the superior trochlear system.

\section{Discussion}

This systematic review summarizes existing literature on potential corridor and vessel options for endoscopic free flap reconstruction of the skull base. Data on this subject are limited to cadaver studies, case reports, and case series.

\section{Pedicle Corridors}

Since most vessels with appropriate vein and arterial caliber suitable for microvascular anastomosis are located in the neck, an appropriate conduit to the skull base needs to be created. This conduit needs to be appropriate in size to minimize kinking and vessel obstruction. In addition, the creation of this corridor should not add significant additional morbidity. As the reconstructive demands and geometry of each surgical defect is unique, it is important for the recon- structive surgeon to be familiar with various options to optimize the surgical outcome.

To date, most data are available on the Caldwell-Luc/ transbuccal corridor. This corridor was initially described in a cadaver dissection. ${ }^{3}$ It was later described in a case report in which a clival defect was reconstructed after a sequestrectomy following osteoradionecrosis. ${ }^{4}$ Our institution later published a series of recalcitrant CSF leak using this corridor with a radial forearm flap. ${ }^{5}$ Since then, we have done an additional four radial forearm flaps successfully delivered to the skull base, and the same corridor was used with myofascial vastus lateralis musculature in another case series. ${ }^{6}$ Creation of this corridor poses some risk of surgical morbidity. Manipulation of the infraorbital nerve may lead to paresthesia in its distribution. In addition, blunt dissection through the buccal space puts facial nerve branches at risk, although this morbidity has not specifically been reported.

Durmaz et al initially described the prevertebral corridor in a cadaver study. ${ }^{7}$ In their description, this corridor was used for delivery of a regional fascial flap based on the occipital vessel for reconstruction of extensive defects of the skull base. The same corridor was later described in a case report, in which a radial forearm free flap was used to reconstruct a clival defect. ${ }^{8}$ This corridor is in the plane just anterior to the prevertebral fascia and offers a direct route to the skull base without traversing critical neurovascular structures; furthermore, dissection in the proper plane can be blunt and quick, and the flap pedicle can run a short and straight course without kinking. Consequently, this approach is an enticing option for the reconstruction of clival or posterior pharyngeal defects.

The transpterygoid/parapharyngeal corridor also allows for a direct route and favorable vessel geometry. In contrast to the prevertebral corridor, this approach uses the space anteromedial to the great vessels. Dissection in the parapharyngeal fat, medial to the medial pterygoid space, will lead to the pterygoid plate just posterior to the maxillary wall. To deliver the pedicle into the sinonasal cavity, the posterior maxillary wall and pterygoid plate must be removed. Unless the pterygoid plates were removed as part of the surgical resection, this approach may lead to additional surgical morbidity in terms of injury to the neurovascular structures within the pterygopalatine fissure and infratemporal fossa. The corridor was described in detail in a cadaver study by Rivera-Serrano et al, ${ }^{9}$ followed by a case report describing a successful delivery of a serratus anterior myofascial flap for reconstruction of a clival defect. ${ }^{10}$ Furthermore, we have successfully used this corridor at our institution on two occasions to reconstruct the skull base without significant morbidity. Of note, the parapharyngeal space can also be used to get access to the upper pharynx without combination of a transpterygoid approach. This mandates a surgical defect in the pharyngeal constrictors or removal of the medial Eustachian tube.

The transmaxillary corridor was described in a single case report by Kato et al. ${ }^{11}$ This approach potentially carries considerable risk, as the pedicle is tunneled across the face in the maxillary subcutaneous tissues. It could be applicable 
in very select situations; however, it should be noted that this approach is represented in a single case report and the authors have not performed this approach at our own institution.

\section{Vessel Options}

Multiple vessel options for free flap reconstruction of the skull base have been described. Many are well known to the reconstructive head and neck surgeon, including the facial vessels, superficial temporal vessels, and superior thyroid vessels. In perhaps the most novel approach, Reyes et al described the ability to achieve tension-free anastomosis with a 7-cm flap pedicle and the supratrochlear artery via a Lynch incision in a cadaver. ${ }^{13}$ However, the authors note that since this is a cadaver study, cosmetic sequelae of a radix osteotomy cannot be assessed, and closure of the osteotomy could potentially compromise the flap pedicle. ${ }^{13}$ Of note, none of the case reports in this review describe the use of vein grafts. While vein grafts can significantly increase the length of a flap pedicle, allowing a flap to reach its recipient position while still being anastomosed in the neck, they do introduce additional risk in an already complex reconstruction.

\section{Study Limitations}

There are several limitations to this study. There is a paucity of published data on endoscopic free tissue transfer for skull base reconstruction, and the majority of studies involve cadavers. While several cases have been described for the Caldwell-Luc/transbuccal space corridor, only one case report each has been published describing the prevertebral, transpterygoid/parapharyngeal, and transmaxillary approaches. Furthermore, no case reports have been published describing the use of the supratrochlear artery or third segment of the maxillary artery as recipient vessels for endoscopic skull base free flap reconstruction. Furthermore, the small quantity of case reports leads to the possibility of a significant publication bias. Also, while the surgical corridors are well described, limited data are available on geometry and pedicle length required to reach the target. As such, many of these approaches may rarely or never be performed in real clinical situations due to anatomical constraints, lack of instrumentation, or technical difficulties.

\section{Future Directions}

More robust data and outcome measures including flap viability and patient morbidity are necessary. Prospective data collection is likely unfeasible, and consequently further data will need to come from additional case reports, case series, and retrospective studies. As the field of endoscopic endonasal surgery continues to expand, the role of free tissue transfer will continue to evolve.

\section{Conclusion}

There are several options available for reconstruction of the skull base for CSF leak or after tumor resection including local and pedicled flaps. In rare cases, free tissue transfer may be required. This review summarizes several described and potential routes for free flap reconstruction of the skull base.

\section{Conflict of Interest}

The authors have no conflicts of interest to disclose.

\section{References}

1 Hachem RA, Elkhatib A, Beer-Furlan A, Prevedello D, Carrau R. Reconstructive techniques in skull base surgery after resection of malignant lesions: a wide array of choices. Curr Opin Otolaryngol Head Neck Surg 2016;24(02):91-97

2 Slim K, Nini E, Forestier D, Kwiatkowski F, Panis Y, Chipponi J. Methodological index for non-randomized studies (minors): development and validation of a new instrument. ANZ J Surg 2003;73(09):712-716

3 Kamal SM, Cho A, Jacobson JP, Shahlaie K, Luu QC. Free flap reconstruction of endoscopic skull base defects. Otolaryngology Head and Neck Surgery (United States) 2012;147:158-P159

4 Hackman TG. Endoscopic adipofascial radial forearm flap reconstruction of a clival defect. Plast Reconstr Surg Glob Open 2016;4 (11):e1109

5 Sinha P, Desai SC, Ha DH, Chicoine MR, Haughey BH. Extracranial radial forearm free flap closure of refractory cerebrospinal fluid leaks: a novel hybrid transantral-endoscopic approach. Neurosurgery 2012; 71(2, Suppl Operative):ons219-ons225, discussion ons225-ons226

6 Kang SY, Eskander A, Hachem RA, et al. Salvage skull base reconstruction in the endoscopic era: vastus lateralis free tissue transfer. Head Neck 2018;40(04):E45-E52

7 Durmaz A, Fernandez-Miranda J, Snyderman CH, Rivera-Serrano C, Tosun F. Prevertebral corridor: posterior pathway for reconstruction of the ventral skull base. J Craniofac Surg 2011;22(03):848-853

8 London NR Jr, Ishii M, Gallia G, Boahene KDO. Technique for reconstruction of large clival defects through an endoscopicassisted tunneled retropharyngeal approach. Int Forum Allergy Rhinol 2018;8(12):1454-1458

9 Rivera-Serrano CM, Snyderman CH, Carrau RL, Durmaz A, Gardner PA. Transparapharyngeal and transpterygoid transposition of a pedicled occipital galeopericranial flap: a new flap for skull base reconstruction. Laryngoscope 2011;121(05):914-922

10 Krane N, Troob S, Wax MK. Combined endoscopic and transcervical approach for free flap reconstruction of nasopharyngeal and clival defects: a case report. Microsurgery 2019;39(03):259-262

11 Kato H, Mizuta K, Yamada N, Ueda N, Ito Y. A new route for passing a free flap vascular pedicle using contralateral facial vessels as recipient vessels in skull base reconstruction. Plast Reconstr Surg 2012;130(01):212e-214e

12 Assam JH, Quinn TH, Militsakh ON. The maxillary artery as a recipient vessel option for complex midface and anterior skull base microsurgical repair: a cadaveric study. Microsurgery 2017;37(06):611-617

13 Reyes C, Solares CA, Fritz MA, Groves M, Bentley H. Fascia lata free flap anastomosed to the superior trochlear system for reconstruction of the anterior skull base. J Neurol Surg B Skull Base 2017;78 (05):393-398 